\title{
Rationale for Implementing Dose Reference Level as a Quality Assurance Tool in Medical Radiography in Nigeria
}

\author{
Dlama Zira Joseph ${ }^{1}$, Obetta Chinedu ${ }^{3}$, Nkubli Favious ${ }^{2}$, Geofrey Luntsi ${ }^{2}$ \\ Laushugno Shem ${ }^{3,}$ Yabwa Dlama ${ }^{4}$ \\ ${ }^{I}$ Department of Radiology Abubakar Tafawa Balewa University Teaching Hospital, Bauchi,Nigeria \\ ${ }^{2}$ Department of Medical Radiography University of Maiduguri, Nigeria \\ ${ }^{3}$ Department of Radiography Nnamdi Azikiwe University, Awka , Nigeria \\ ${ }^{4}$ Physics Department, Taraba State universty, Jalingo, Nigeria \\ Corresponding Author: Dlama Zira Joseph \\ Email: josephdlama@gmail.com.
}

\begin{abstract}
:
Background: There has been a number of approach to dose reference level used for medical imaging in Nigeria, to facilitate standardization and ensure optimization for dose reference levels in Medical radiography, specific protocols were reviewed to give detailed inter and intra hospital variations for the same radiologic examinations as recommended by international organizations such as International Atomic Energy Agency, International Commission on Radiological Protection and Committee for European Commission.

Objective: To provide protocols for setting dose reference level as quality assurance tool for optimization in medical radiography.

Methodology: This paper is a conceptual review that suggests a concise and comprehensive method for modeling radiodiagnostic dose survey in Nigeria. The work provides adequate information consistent with the Medical imaging task which justifies and provides sufficient ground for setting a protocol for dose reference level.

Conclusion: This paper promotes the nitty-gritty of setting dose reference level as a quality assurance tool in medical Radiography.
\end{abstract}

Key Words: Entrance skin dose, protocols, Diagnostic, Radiography, exposure.

\section{Introduction}

The term Diagnostic reference level or reference value sets an investigation level to identify unusually high radiation dose or exposure levels for common diagnostic medical X-ray imaging procedure. $[1],[2],[4]$. Diagnostic reference levels, provides a practical system that allows hospitals (Radiology Department) to compare their radiation doses delivered to patients. To achieve this, the dose must be expressed in terms of dose quantities that are clearly defined and can be calculated from readily available exposure parameters ${ }^{[5],[6],[10]}$. The dose reference level is not Universal but specific to a country, region, equipment and procedure ${ }^{[11],[14]}$. The need for patient dose measurement has been emphasized in national protocol for dose measurement in diagnostic radiology by NRPB in collaboration with institute of physical science in medicine and college of radiographers. United Kingdom ${ }^{[14],}{ }^{[15]}$.Diagnostic Radiography examination play an important role in the health care of the population in Nigeria and world Wide. These examinations may involve significant irradiation of the patient and probably represent the largest manmade source of radiation exposure for the population ${ }^{[1],[3],[5]}$. X-ray is said to be the major contribution to the collective effective dose of the general public (Personnel and patient) ${ }^{[3],[15],[30]}$

The need for radiation dose assessment of the patient during diagnostic X-ray examination has been highlighted by increasing knowledge of hazard of radiation protection. ${ }^{[19], ~[25]}$

Exposure to ionizing radiation carries with it an increased risk of malignant disease and a risk of hereditary disease in descendants of exposed person $[8],[10],[20]$. There is also the possibility of inducing deterministic effects, however the overall benefits from the diagnostic use of ionizing radiation in medicine greatly exceeds the small risk to the individual from the radiation exposure ${ }^{[4]}$. Moreover, wide variations in patient dose for the same type of X-ray examination has been evident from various dose surveys ${ }^{[3],[13],[22],[23]}$. This variation indicated that a good imaging technique was necessary to reduce patient doses to the lowest practicable levels consistent with the clinical purpose of medical examination.

The National Radiation Protection Board (NRPB) in the U.K published the result of the Nation Wide Survey for a selection of X-ray examination in 20 U.K Hospitals ${ }^{[22],[23]}$. They found that there was a ratio of almost 50 between the hospital with the highest dose and that with the lowest dose for an average sized patient. 
A similar Nationwide survey by the Food and drug Administration of U.S.A revealed that the ratio of the maximum to minimum exposures ranged from 8.8 to $126.7^{[23],[24] \text {. }}$

The patient dose survey provide important information on the level of patients exposure and provide an insight into causes of their variation, radiographic procedures, patients attributes, technical and equipment factors, exposure parameters and the level of quality assurance in place. Considerable evidence shows that significant reduction in radiation dose delivered to patients are possible without detriment to patients care ${ }^{[23]}$.

Both international Commission on Radiological protection (ICRC) and international Atomic Energy Agency (IAEA) recommends the use of dose reference levels in Radiology [6],[11], [16], [33]

The aim of this research paper is to provide guidance document and protocol to regional, National and Local authorized bodies and the clinical community on the application of dose reference level as a quality assurance tool in diagnostic (Medical) radiography.

\section{The Review}

\section{Role Of Relevant Authority (NNRA And NIRPR) In DRLs}

Establishing a National DRLs should be for particular practices and equipments. Relevant Authority such as Nigeria Nuclear Regulatory Authority (NNRA) and National institute for Radiation Protection and research are saddled with the responsibilities of research and training of radiation protection personnel (RPP) in Nigeria. In view of the need to establish the NDRLs in Nigeria and the significant roles played by parallel bodies in the UK (National Radiological Protection Board, Royal college of Radiologist) and in US (American College of Physicist in Medicine and American College of Radiologist) in establishing NDRLs ${ }^{[30]}$.

The parallel bodies responsible for this in Nigeria are NNRA and NIRPR. They are expected to be responsible for periodic review to ensure dose optimization which is possible by carrying out regular dose audit and inculcating NDRLs programme into the accreditation of health institutions using ionizing radiation ${ }^{[30]}$

There is a need for National Radiation Dose Database (NRDD) and Audit to access dose information and easy processing of the same for future optimization programme. However a detailed and comprehensive data should be collected by funding researchers at Masters and PhD level in that regard ${ }^{[30]}$

\section{Adopted Dosimetric Parameters For DRLs}

DRLs involves all the procedures that is employed during radiological procedures and medical imaging in Radiology Department. They include Conventional x-rays, fluoroscopy procedures, dental X-rays, computed tomography and angiography examinations. However, computed tomography over the years have gain recognition in Nigeria and it delivers higher radiation doses to patients of up to $20 \mathrm{mSv}$ and radiation induced cancer risk of up to 1 in 1000 per examination ${ }^{[25],[30],[35]}$.

Hence, there is urgent need of DRLs in Nigeria and all other developing countries in Sub-saharan Africa. In the United Kingdom, reference doses are based on 75 percentile, which are values of the distribution of the mean dose observed in Many Hospitals. ${ }^{[12],[35]}$. Meanwhile in United State the DRLs are based on 80 percentile of the survey distributions doses without backscatter. ${ }^{[13]}$

In the UK and US equipments using higher radiation doses than $75 \%$ and $80 \%$ respectively require correction measures because the reference level doses corresponds to the $75^{\text {th }}$ percentile of individuals that receive doses less than this value. This implies that dose reduction should be possible for the $25 \%$ of individual whose dose exceed the guidance level ${ }^{[15]}$.

\section{Patient Dosimetric Parameter}

Some adopted dosimetric parameters that are widely used includes ${ }^{[30]}$

1. Entrance Surface Dose (ESD): Conventional radiography (could be obtained with TLD, instant dose or by calculations.)

2. Dose Area Product (DAP): Conventional Radiography and fluoroscopy (obtained with DAP meter)

3. Weighted Computed Tomography Dose Index $\left(\mathrm{CTDI}_{\text {index }}\right)$ per slice in serial CT scanning or per rotation in helical CT scanning.

4. Dose Length Product (DLP) per complete CT examination.

However, three quantities play central role in radiation dosimetry; these are Kerma, ESD and effective dose (E).

A dose reference protocol has been suggested ${ }^{[25]}$

1. Survey of Hospital equipments

2. Measuring free Air Exposure (FAE)

3. Converting FAE to ESD

4. Setting DRL: Dose Audit for Local DRLs and National DRLs and compare with International DRLs 


\section{Survey of hospitals and equipments}

In establishing Dose reference levels both at Local and National level it is important to survey as many hospitals as possible. Both at primary and Tertiary Health Care Level including private hospitals across the geopolitical zones and states in the country. The x-ray machine, its technical factor and exposure data should be properly and carefully surveyed ${ }^{[2],[4],[5],[9] .}$

The hospital equipment and the radiographers who operate X-ray machines determine the output of the exposure delivered. The need for standardization of radiation exposure and guidance levels for various radiographic examinations has also been proposed by the international Atomic Energy Agency (IAEA) as a safety standard. ${ }^{[15],[25] \text {. }}$

The X-ray machine usually consist of X-ray tube, beam restrictor (collimator) and in many cases a grid. Each of these components of X-ray machine is to be taken consideration in surveying Dose reference levels.

A sample of dose survey form has been recommended in dose reference levels ${ }^{[25]}$. In today's diagnostic radiology, there is a growing concern about radiation exposure. This can be seen in the recommendations of ICRP ${ }^{[18],[19],[20] .}$

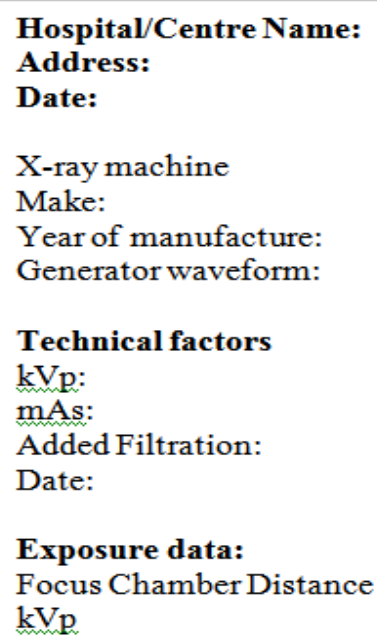

\section{Measuring Free Air Exposure (FAE)}

One of the important routine QC test that is usually constructed in each X-ray machine is the output (FAE) accuracy at different $\mathrm{kVps}$. This test must yield a straight line relationship between $\mathrm{kVps}$ and output (FAE). The output Vs kVps relation should be measured for each X-ray Machine used.

Using the ESD criteria to set up DRL requires accurate measurement of the quality. However the ESD can be measured directly using thermoluminiscent dosimeters and indirectly by the use of Dose Area Product (DAP) meter and ion chamber to measure free Air Exposure (FAE) ${ }^{[8],[10],[25]}$

TLD is widely used in Nigeria to asses ESD due to their sensitively and tissue equivalent but there are few TLD readers in the country which hinders and limits the use of TLD for National Survey. The use of DAP meters is limited by the fact that dose and area cannot be separated ${ }^{[25] .}$ However the FAE at the point where the $\mathrm{X}$-ray central beam measures strike the body may be measured using ionization chamber. ${ }^{[4],[20],[26],[27] .}$

Standard methods for measuring the FAE for manual and automatic exposure control units have been described by the American Association of Physicist in Medicine (AAPM) ${ }^{[2]}$. In the AAPM protocol, the ion chamber is placed approximately above the table top to minimize back scattering. Back scatter factor is almost needed in each formula of ESD. The BSF was modeled from the data published for different X-ray machines and field sizes. ${ }^{[14],[15] .}$ Some of the ESD calculation methods require knowledge of the half value layer (HVL) of different machines at different $\mathrm{kVp}$ setting are not usually performed during quality control processes and instead the HVL is usually measured at a constant $\mathrm{kVp}(90 \mathrm{kVp})$ in most cases. All these parameters can be obtained from the survey forms when properly filled by the hospitals.

\section{Converting FAE To ESD}

To save time and equipment FAE could be measured at a particular set of parameters say $80 \mathrm{kV}$, mAs, and at a distance of $100 \mathrm{~cm}^{[25]}$ 
Correction for other parameters used for the real patient will then be made using the formula ${ }^{[12]}$. $\mathrm{FAE}(\mathrm{mR})=$ tube Output $\mathrm{x}\left(\mathrm{kV}^{2} / 80^{2}\right)\left(100^{2} / \mathrm{FSD}^{2}\right) \times \mathrm{mAs}$.

Where tube output is FAE at $80 \mathrm{KV}$ at a distance of $100 \mathrm{~cm}$ normalized by $\mathrm{mAs}(\mathrm{mR} / \mathrm{mAs}) \mathrm{kV}$ is the tube potential, mAs is the product of the tube current and exposure time and FSD is the focus to skin distance. The FAE above is defined free in air without any back scatter. To obtain the ESD to air with back scatter the following formula could be used.

\section{$\mathrm{ESD}(\mathrm{mGy}) \quad=$ FAE $(\mathrm{mR}) \times 0.00877 \times \mathrm{BSF}$}

Where the constant 0.00887 converts the FAE in $\mathrm{mR}$ to ESD in $\mathrm{mGy}$ and the back scatter factor (BSF) accounts for contribution from backscatter radiation. The BSF depends on the factors such as $\mathrm{kV}$, field size, FSD

\section{Setting DRL}

After obtaining detailed and adequate information from 1 to 3 above. It is necessary to analyze and record the information obtained. The analysis should be based an ESD and technical parameters ${ }^{[25] .}$ The third quartile of the ESD would then be taken as Dose Reference Level for each examination included in the survey ${ }^{[35]}$

\section{Radiation Safety Committee (RSC)}

RSC must be constituted as an effort of Management Commitment to ALARA "As Low as Reasonably Achievable" with economic and social factors taking into consideration. RSC designates authority to the radiation safety office for enforcement of ALARA concept and will support Radiation Safety Officer (RSO) when it is necessary for Radiation Safety Officer to assert Authority. A formal review of radiation safety program is performed annually and this includes reviews of the operating procedures and keeping record of Dose reference Levels as a result of Monitoring radiation to patients and personnel by using appropriate dosimeter where necessary. All these must be based on technology and economic considerations. RSC should also carry out dose Audit for LDRLs and compare with NDRLs and international DRLs

\section{Conclusion}

There is a need for professionals to stay abreast with various approaches that has been taken by authorized bodies working in concert with professional working groups in other to help manage radiation dose to patients so as to ensure that patient dose is commensurate with the clinical purpose. DRL is a protocol to be implemented at Regional, National and Local Levels. Radiation safety committee is very vital in ensuring safety and setting protocols for DRLs in Diagnostic Radiography. The radiation safety officer is recommended for each hospital in setting Local DRLs.

\section{References}

[1]. Ajayi I.R., and Akinwumiju A.A. measurement of entrance skin dose to patients in four common diagnostic examinations in Nigeria. Radiation Protection Dosimetry. 2000, 87:217-220.

[2]. American Association of Physicist in Medicine, AAPM (1991). Report 31,standard methods of measuring diagnostic x-ray exposures. Medical physics publishing, Madison.

[3]. Babalola, I.A. 2004. "In Quest of Light Quanta". University of Ibadan Inaugural Lecture Series. Conference on Radiation Control Program Directors, CRCPD (1988). Average patient exposure guides, CRCPD publications, 88-85.

[4]. Contento G., Malisan M.,and Padovani R. (1988) a comparison of diagnostic radiology practic and patient exposure in Britain, France and Italy.British journal of radiology, 61: 143-53.

[5]. CEC, Commission of European Communities. 1990. "Quality Criteria for Diagnostic Radiographic Images". Working document CEC XII/173/90, 2nd edition. Commission of the European Communities: Brussels, Belgium.

[6]. CEC. 1996. "European Guidelines and Quality Criteria for Diagnostic Radiography Images". Eur 16260 EN. The European Commission: Brussels, Belgium.

[7]. CEC. 1997. "Council Directive on Health Protection of Individual against Danger of Ionizing Radiation in Relation to Medical Exposure and Replacing Directive 84/466/Euroatom”. The European Commission: Brussel, Belgium.

[8]. Compagnone, G., Pagan, L., and Bergamini, C. 2005. "Effective Dose Calculation in Conventional Diagnostic X- Ray Examinations for Adult and Pediatric Patients in a Large Italian Hospital”. Radiation Protection Dosimetry. 114(1.3):164 - 167.

[9]. Curry TS, Dowdey JE, Murry RC: Christensen's Introduction to the physics of Diagnostic Radiology. 3rd ed. Lea \& Febiger, Philadelphia, PA, 1984.

[10]. Davies, M., McCallum, H., White, G., Brown, J., and Hlem, M. 1997. "Patient Dose Audit in Diagnostic Radiography using Custom Designed Software". Radiography. 3:17-25.

[11]. European Commission (EC) Council Directive 97/43/Euratom of 30 June 1997 on healthprotection of individuals against the dangers of ionizing radiation in relation to medical exposure.

[12]. Faulkner K, Broadhead D.A, and Harrison R.M. (1999) Patient dosimetry measurement methods. Applied Radiation and Isotopes 50, 113-123.

[13]. Gray J.: Reference Values - What are they? American Association of Physicist in Medicine. (AAPM). Newsletter 24, 9-10, (1999), Medical Physics Publishing, Madison.

[14]. Hart D, Hiller M, and Wall B. (NRPB 2002). Doses to patients from medical x-ray examination in the U.K. 2000 review NRPBW14.

[15]. Hart, D., Hillier, M. C., Wall, B. F., Shrimpton, P. C., and Bungay, D. (1996). Doses to patients from medical X-ray examinations in the UK, 1995 review. NRPB Report R289. NRPB, Chilton. 
[16]. International Atomic Energy Agency (IAEA). (1995). Radiation doses in diagnostic radiology and methods for dose reduction. IAEA-TECDOC- 796, Brussels; CEC.

[17]. International Commission on Radiological Protection, ICRP :1990 Recommendations of the ICRP, ICRP pub.60, (1991), Pergamon Press Oxford. CRP. 1990.

[18]. "Recommendation of the International Commission on Radiological Protection”. ICRP Publication 64. Ann ICRP 1991. 21 (1-3). Pergamon Press: Oxford, UK.

[19]. 18. IPEM/ NRPB/RCR/BIR. 2000. "The Newsletter of the Institute of Physics and Engineering in Medicine". Publication, No. 67. IPEM: New York, NY.

[20]. Johnston and Brennan. 2000. "Reference Dose Levels for Patients undergoing Common Diagnostic X-Ray Examination in Irish Hospitals". Br. J. Radiol. 73: 396-402.

[21]. National Council on Radiation Protection and Measurement. (1987): NCRP Report No. 93, "Ionizing Radiation Exposure of the Population of the United States" NCRP, Bethesda, Maryland.

[22]. James, EM. (2006). Physics of Radiation Protection. 2nd Ed. Wiley-VCH Verlag GmbH \& Co. Weinheim. Pg 348.

[23]. National Radiological Protection Board (NRPB) / Royal College of Radiologist. (1990): Patient Dose

[24]. National Radiological Protection Board, NRPB (1993): "Radiation Exposure of the U.K Population" NRPB Report R263- 1993 Review. NRPB, Chilton, U.K.

[25]. Ogundare,, F. O.; Olarinoye, I.O. and Obed, R. I..(2008). Estimation of patients' organ doses and conceptus doses from selected Xray examinations in two Nigeria $\mathrm{X}$-ray centres.

[26]. Olarinoye, I. O., and Sharifat, I. a protocol for setting dose reference level for medical radiography in Nigeria: a review Bayero Journal of Pure and Applied Sciences, 3(1): 138 - 141.(2010) 26. Radiation Protection Dosimetry 2008 132(4):395-402

[27]. Ogundare F.O., Ajibola C.L., and Balogun F.A. (2004a). Surveys of radiological techniques and doses of children undergoing some common X-ray examinations in three hospitals in Nigeria.Medical Physics 31(3). 521-524

[28]. Ogundare, F.O.; Uche, C. Z.; Balogun F.A. ; (2004b) Radiological parameters and radiation doses of patients undergoing abdomen, pelvis and lumbar spine $\mathrm{X}$-ray examinations in three Nigerian hospital. British journal of radiology, 77, 934-940.

[29]. Ogunseyinde, A.O.; Adeniran, S.A.M.; Obed,R.I.; Akinlade, B.I.; Ogundare, F.O. (2002). Comparison of entrance surface doses of some X ray examinations with CEC reference doses. Radiation. Protection Dosimetry. 98, 231-234.

[30]. Olowookere, C.J., I.A. Babalola, N.N. Jibiri, R.I. Obed, L. Bamidele, and E.O. Ajetumobi. 2012. "A Preliminary Radiation Dose Audit in some Nigerian Hospitals: Need for Determination of National Diagnostic Reference Levels (NDRLs)". Pacific Journal of Science and Technology. 13(1):487-495.

[31]. Oyeleke O.I. (2009). Entrance Skin Dose (ESD) Evaluation for Pediatric Chest Examinations in Minna and Ibadan. Journal of science, education and technology, 2(1). 146-150 [31]

[32]. Sharifat, I. and Olarinoye I.O. (2009). Patient Entrance Doses at Minna and Ibadan for Common Diagnostic Radiological Examinations. Bayero Journal of Pure and Applied Sciences. 20092 (1): 1-5.

[33]. The institute of physical Sciences in Medicine (IPSM), NRPB and College of radiographers (CR): National protocol for patient dose measurements in diagnostic radiology. NRPB, Oxon, 1992.

[34]. Tung C.J, Tsai H.Y, and Lo S.H. (2001).Determination of guidance level of dose for diagnostic radiography in Taiwan. Medical Physics 28 (8), 850-857.

[35]. Wall B.F and Shrimpton P.C. (1995). Patient dose protocol and trend in the U.K. Radiation protection Dosimetry, 57:359-362. 